\title{
PRIMARY CHRONIC INFLAMMATION OF THE CYSTIC DUCT
}

\author{
G. LABò, M.D. \\ L. Rosa, M.D. \\ Cattedra di Semeiotica Medica, University of Bologna, Italy.
}

(Director: Prof. G. Labò)

\begin{abstract}
WE WILL relate observations made during the last ten years on primary chronic inflammation of the cystic duct: these observations will concern only a few aspects of this interesting chapter in biliary pathology, long well known, but on whose theoretical and clinical meaning no full agreement has yet been reached.

When we refer to the cystic duct, we include also the neck and the infundibular portion of the gall bladder, which we consider an anatomical unity.
\end{abstract}

Primary chronic inflammation of the cystic duct is, in our opinion, more frequent than generally supposed. The lack of pathological reports can be ascribed to the reluctance of surgeons to remove gall bladders which do not show gross abnormalities on X-ray examination, and the tendency to consider this condition as a mere functional disorder of the biliary tract.

Our first observations were surgical surprises: radiologically normal gall bladders, which ached with an intensity that we thought due to the presence of stones, appeared instead on microscopic examination as obstructive, hypertrophic, chronic cystic duct inflammations, with no changes in the gall bladder itself or only minor ones.

These findings induced us to study the problem further. We first tried to define better this clinical-pathological entity with researches into those clinical and laboratory signs that would differentiate this form from chronic cholecystitis and cholelithiasis.

As a matter of fact, since cystic duct inflammation may present all the clinical features of other gall bladder diseases, the differential diagnosis by clinical signs presents remarkable difficulties.

Furthermore, a primary cystic duct inflammation may arise at the same time as a chronic diffuse gall bladder inflammation or be the initial stage of it; or it may also be associated with cholelithiasis or with cholesterosis of the gall bladder. Usually, symptoms are subordinate to the gall bladder reaction to the cystic duct obstruction: they are highly painful, as in cholelithiasis, when the gall bladder is tonic or hypertonic, that is when there is a conflict between the force of contraction of the gall bladder and the obstacle to the evacuation of bile; and dyspeptic, when the gall bladder is hypotonic.

Between these two forms (painful and dyspeptic) there exist others, such as the pseudoulcerous, which reveal the reflex and inflammatory gastro-duodenal component of biliary pathology.

In colic caused by isolated primary cystic duct inflammation, the inflammatory signs, which characterize the biliary colic of chronic cholecystitis and cholelithiasis, are usually not observed, while the slight paroxysmal pains localized to the epigastric or to the subhepatic region, which appear after meals or during menstruation, after physical or emotional stress, are usually present.

$\mathrm{X}$-ray examination reveals evidence both direct and indirect, while in cases of total obstruction of the cystic duct the gall bladder is not filled.

The direct features are the morphological alterations of the cystic duct (thread-like duct, rigid duct, rosary-like duct or in the form of a swan-neck); equally important and more frequent is the indirect evidence, such as the delay in the gall bladder visualization time, the tonichypertonic aspect with often rapid and efficient evacuation (sometimes only partial or absent) and a large, hypotonic, gall bladder. Laboratory findings on bile can also supply important evidence. We study, as a rule, the gall bladder concentrating power by evaluating iodine content of the cholecystic bile after intravenous injection of iodated radiopaque substance. No changes in this concentration are observed in the primary chronic cystic duct inflammation; while they are present in diffuse chronic cholecystitis.

Electrophoretic studies on bile, according to techniques long-tested in our department, have supplied other important data; among which is the identification of typical inflammatory 


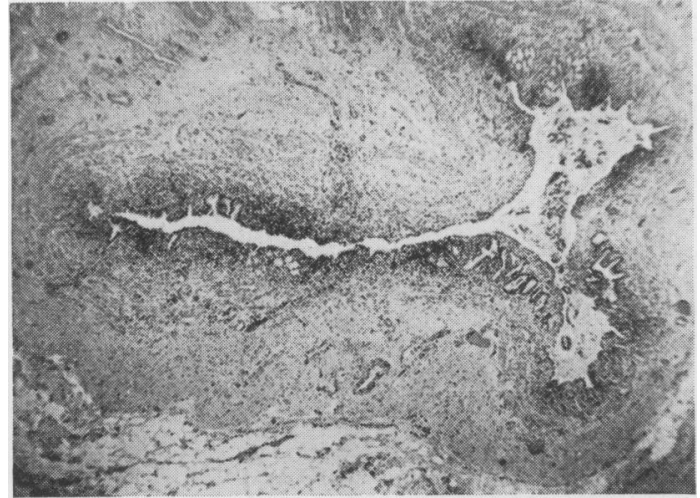

FIG. 1.-Atrophic chronic cysticitis. The width of the cystic duct, in oblique section, appears reduced The mucous membrane is flattened, deficient of epithelium and densely infiltrated by round cells. In the basement membrane are bundles of connective tissue, between the muscular fibres. (H. \& E. x200).

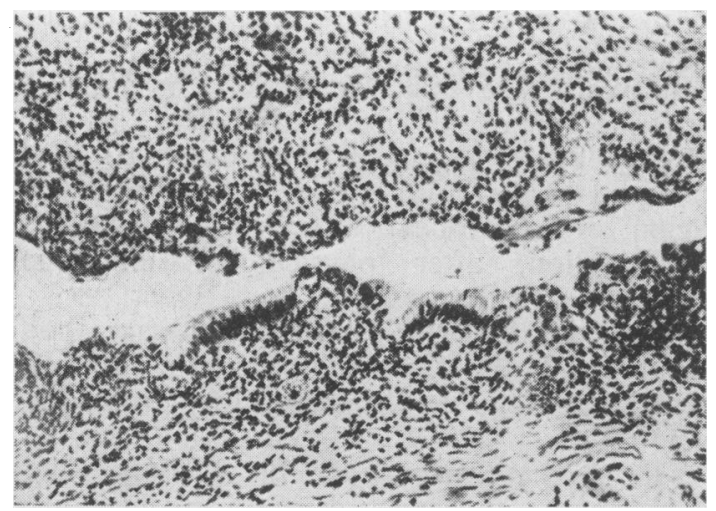

FIG. 2.-Atrophic chronic cysticitis. Detail of Fig. 1. (H. \& E. x200).

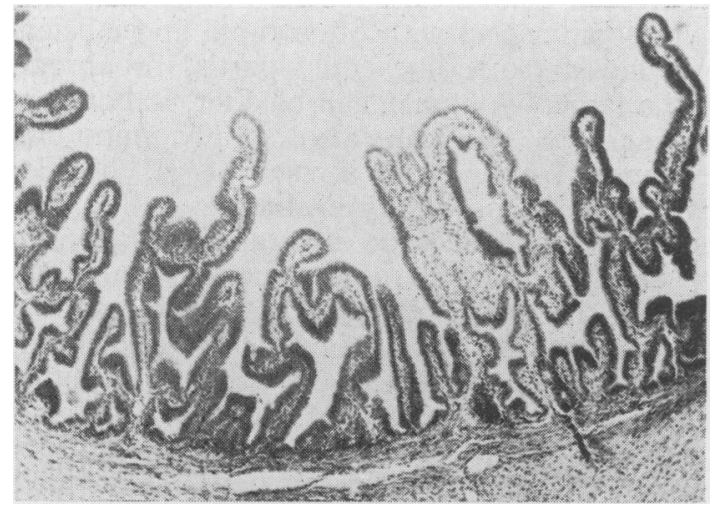

Fig. 3.-Normal gall bladder. Same case as in Fig. 1 and 2 (H. \& E. x80).

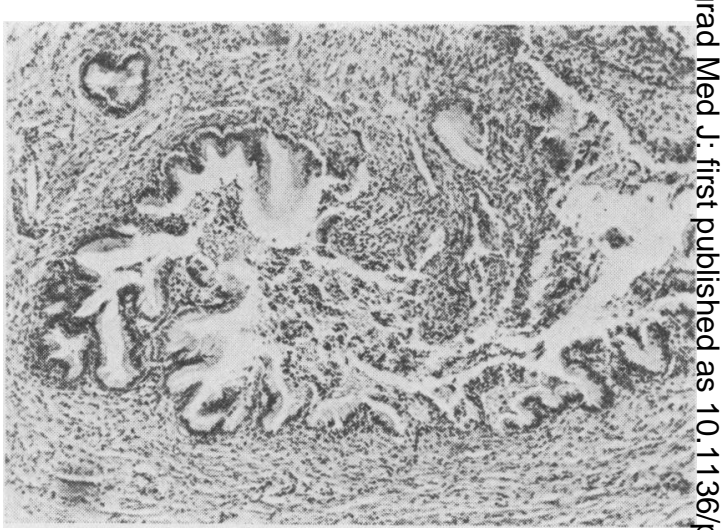

FIG. 4.-Hypertrophic chronic cysticitis. The width of the cystic duct is narrowed by large folds of mucous membrane. (H. \& E. X80).

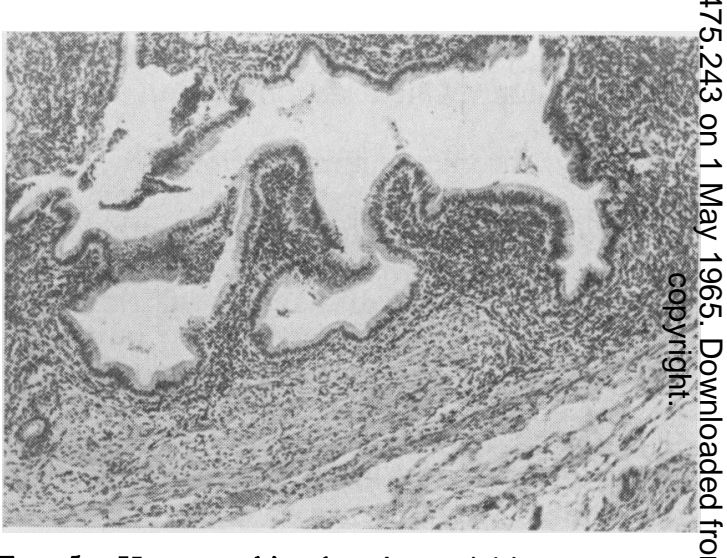

FIG. 5.-Hypertrophic chronic cysticitis. Large folds of mucous membrane with a conspicuous infile tration of neutrophils around their axes, project forth in the duct. The muscular fibres are separated by large connective tissue bundles? (H. \& E. x80).

syndromes of chronic cholecystitis (with or with응 out stones) characterized by the increase of pros tein and glycoprotein materials, with particula? increase of the part with the most rapid migra tion; non-inflammatory syndromes, which we have defined as dysmetabolic, pure lithiasiso diathesis, are characterized by an increase of sudanophilic material and in particular of an electrophoretic inert component, composefo mainly of cholesterol.

The primary chronic cystic duct inflamma tions are characterized, instead, by an almos normal electrophoretic pattern.

Other diagnostic elements are offered by the study of the biliary sediment and of the activat ing capacity of bile on lipase. The former 


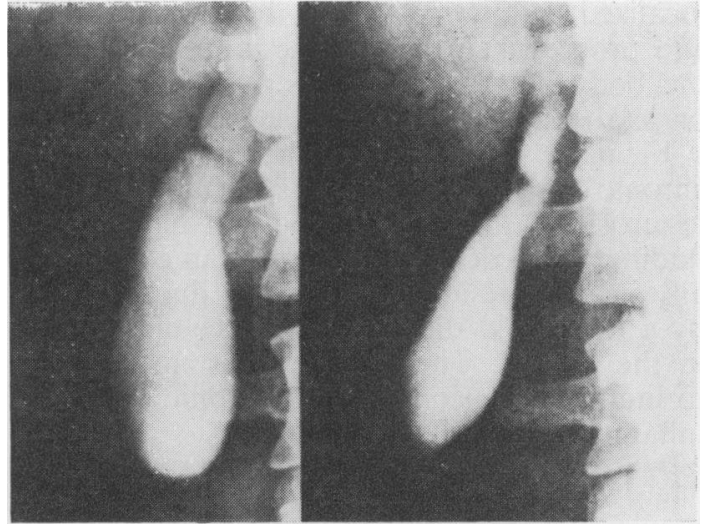

FIG. 6.-Cholecystography in a case of cystic duct and cholecyst cholesterolosis. The gall bladder, in the shape of a sausage, is constricted by an infundibular spasm. The gradation of the shadow is normal (A). After stimulation (B), a delayed contraction, with an increase of the infundibular spasm, is perceived. Therefore we are here observing a hypotonic cholecystostasis associated with alterations of the cystic-infundibular region.

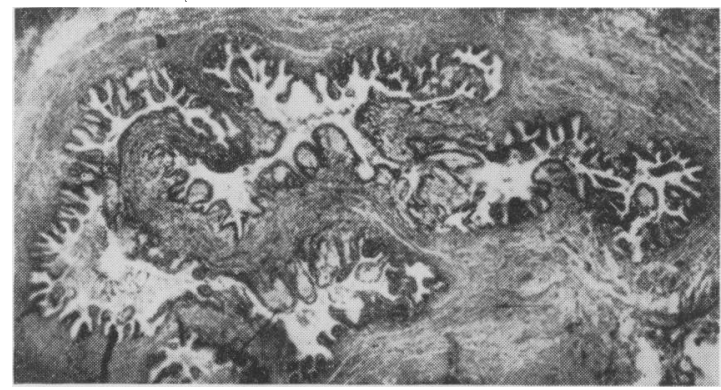

Fig. 7.-Cystic duct cholesterolosis. Same as in Fig. 6. Mucous folds, projecting forth in the cystic duct and narrowing its width, are observed. Underneath the epithelium, we observe groups of histiocytes, containing lipoids. In the deepest mucous layers sclerosis is present. (H. \& E. X15).

shows a bile with some inflammatory elements, the latter is normal.

The anatomical study of chronic bile-duct infection has furnished data of great theoretical and practical importance, not only in these inflammations in particular but also in regards to the pathogenesis and evolution of the lesions.

There is almost always a noticeable disproportion between a bladder wall that is almost normal and the thickening of the cystic duct, which can extend to the neck and infundibulum of the gall bladder.

Histologically, in the initial stages we have predominantly œdema, desquamation and necrosis of the mucosa. This is followed by a

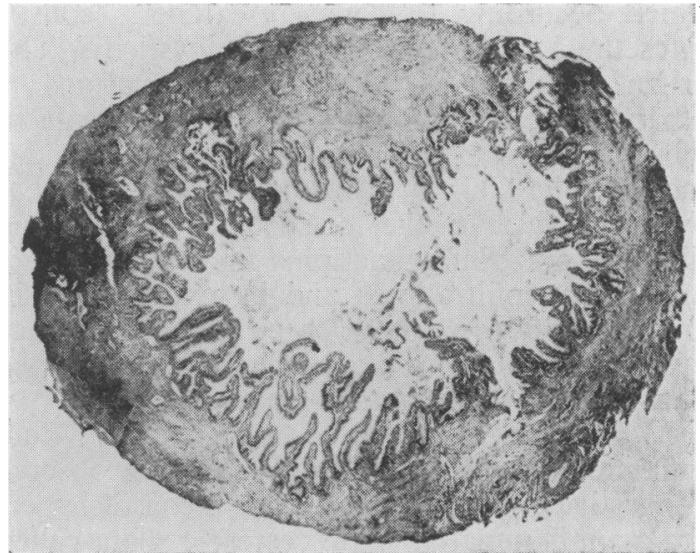

FIG. 8.-Hypertrophic chronic cysticitis with fibrous pericysticitis. Cystic duct section, showing remarkably thickened walls and fibro-sclerotic changes. (H. \& E. x15).

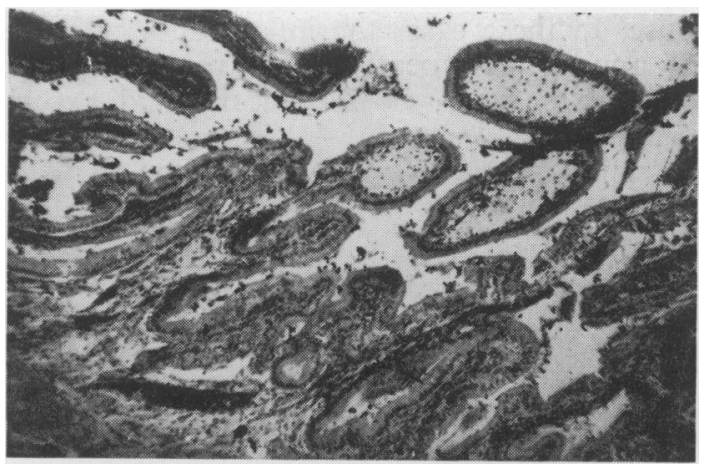

FIG. 9.-Strawberry gall bladder. Same case as in Fig. 8. Large amount of folds, club-like, with heaps of histiocytes containing lipoids. Coat of epithelium normal. (H. \& E. X80).

round-cell infiltration which replaces the initial neutrophil infiltration; the proliferation of the connective tissue, which leads to sclerosis and permanent thickening of the wall, constitutes the final phase.

Moreover, the chronic inflammatory process stimulates a hyperplastic alteration of the mucosa, which leads to a restriction or a complete obstruction of the cystic duct by the forming of big hypertrophic mucosa folds (hypertrophic cysticitis).

Besides those forms, which are more frequent, we observe also a primary atrophic change, which is characterized by the thinness of the mucosa and the lack of epithelium.

Frequently we find fibrous pericysticitis 
which according to some researchers, constitutes the initial stage of chronic cysticitis. The origin of these cystic duct inflammations is attributed to a chronic low-grade bacteremia in which factors of local sensitization play a fundamental role. This is confirmed by the fact that we can observe the coexistence of chronic fibrosclerotic inflammation, foci of necrosis and recent infiltrations; and by experiments in animals, since we have been able to induce cysticitis and chronic cholecystitis by the Sanarelli-Schwartzmann reaction.

Moreover, Albot has described cystic duct inflammations in which it is possible to observe congestive and edematous phenomena of hyperergic inflammatory processes: giant-celled sclero-necrotic cysticitis.

Beside the purely inflammatory forms, there are also primary dysplastic types, in which the obstruction of the cystic duct is secondary to hyperplasia of the mucosa, as it can be observed in dysendocrine states, especially in hyperfolliculinism, which is the determining cause. In these cases the inflammatory process can appear subsequently. We have studied these forms extensively, both clinically and experimentally.

Primary chronic cysticitis seldom remains localized to the cystic duct, but extends slowly and progressively toward the infundibulum and $z$ the body of the gall bladder, by disseminated $\stackrel{\perp}{\stackrel{2}{ }}$ parietal lesions (Albot).

Finally, we consider the possibility that $\Rightarrow$ chronic cysticitis may be associated with cholesterosis of the body of the gall bladder. Our findings have demonstrated that an experiment- $\frac{5}{\omega}$ ally induced lesion of the cystic duct, followed $\vec{\nabla}$ by a hyperlipid diet, may produce cholesterosis 2 in the animal. The diet alone is not sufficient is to induce the lesion, nor the chronic cystic duct $\vec{\circ}$ inflammation by itself.

\section{REFERENCES}

Albot, G., Olivier, C., Libaude, H., and Dressler, H. (1950): Les véscicules Fraises sont des Cysticites $\vec{A}$ Compliquées de Lipoidose Vesciculaire, Bull. Soc. A méd. Paris, 67, 140.

Albot, G., Olivier, C., Poilleux, F., and Libaude, or H. (1950): Les Formes de Début des Cholécysticites Chroniques non Lithiasiques. p. 3. Paris: Vigor.

Albot, G., Pollleux, F. (1952): Les Voies Biliaires. ㄱ Paris: Masson.

Albot, G., Toulet, J., and Pineau, P. (1952): Cys- $\bar{z}$ ticite Fibreuse et Dyskinésie Cystique. Résultats Concordants de la Cholécystographie de Face et de Profil, du Tubage Minuté et de la Radiomano- $\vec{\emptyset}$ métrie Biliaire, Actualités. hépato-gastro-entérg. of Hôtel-Dieu, 50-52. 